The four roots are

$$
P=\frac{d z}{d r}=\frac{\tau \pm K}{\xi+\eta\left(1 \pm i 3^{1 / 2}\right) / 2}
$$

where the \pm signs are independent; the yield equation in the original form of Eq. (5c) has been used in obtaining (12).

4. Conclusion. It is clear that real characteristics do not exist in general, since $P$ can be real only when $\eta=\sigma_{\theta}-\omega=0$. But when $\eta=0$ and $u \neq 0$ the stress equations become identical with the three equations for plane strain, under the Mises yield condition; the characteristic parameter $P$ then reduces to

$$
P=\frac{\tau \pm K}{\xi}
$$

As may be easily verified, this is the standard result for the slope of the characteristic curves in problems of plane plastic flow ${ }^{4}$, which provides a useful check on the present results.

An analogous but simpler investigation of Eqs. (6) leads to the conclusion that there are also no curves on which the velocity derivatives are undefined, even though the values of $u, w$ and the stresses are defined.

${ }^{4}$ See, for example, William Prager, Plasticity for the aerodynamicist, J. Aero. Sci. 15, 253-262 (1948).

\title{
EFFECT OF HYPERBOLIC NOTCHES ON THE STRESS DISTRIBUTION IN A WOOD PLATE*
}

\section{By C. BASSEL SMITH, University of Florida}

1. Introduction. If from an orthotropic material a flat plate is cut parallel to a plane of elastic symmetry, it will have two perpendicular axes of symmetry in the plane of the plate. Such a plate is said to be orthotropic. ${ }^{1}$ An example of this kind of plate is a plain-sawn board. In the discussion that follows, an orthotropic plate is assumed to be bounded on two sides by hyperbolic notches given by the equation $y^{2} / a^{2}-x^{2} / b^{2}=1$, and indefinitely extended in the other direction. The $x$ - and $y$-axes are taken parallel to the axes of symmetry. The plate is subjected only to forces directed parallel to the $x$-axis and applied in the plane of the plate. These forces are assumed to act at great distances from the $y$-axis, and in such a way that the traction over any cross-section perpendicular to the $x$-axis is statically equivalent to a single force of magnitude $P$ directed along the $x$-axis. The problem will be treated as one of plane stress.

2. The stress distribution in a wood plate with hyperbolic notches. The components of stress and strain in the orthotropic plate described are connected by the following relations ${ }^{2}$ :

${ }^{*}$ Received June 11, 1948.

${ }^{1}$ A. T. Price, Phil. Trans. (A) 228, 1-62 (1928). H. W. March, Physics, 7, 32-41 (1936). U. S. Forest Products Laboratory Reports Nos. 1300, 1301, 1304, 1312, 1316.

1. ' ${ }^{2}$ U. S. Forest Products Laboratory Report No. 1503. Love's notations for the stress and strain components are used, (A. E. H. Love, The mathematical theory of elasticity, 4th ed., Cambridge University Press, Cambridge, 1927). 


$$
\begin{aligned}
& e_{x x}=\frac{1}{E_{x}} X_{x}-\frac{\sigma_{y x}}{E_{y}} Y_{y}, \\
& e_{y y}=-\frac{\sigma_{x y}}{E_{x}} X_{x}+\frac{1}{E_{y}} Y_{y}, \\
& e_{x y}=\frac{1}{\mu_{x y}} X_{y} .
\end{aligned}
$$

In these equations $E_{x}$ and $E_{y}$ are Young's moduli in the $x$ - and $y$-directions, respectively. Poisson's ratio $\sigma_{x y}$ is the ratio of the contraction parallel to the $y$-axis to the extension parallel to the $x$-axis associated with a tension parallel to the $x$-axis and similarly for $\sigma_{y x}$. The quantity $\mu_{x y}$ is the modulus of rigidity associated with the directions of $x$ and $y$.

Since the equations of equilibrium are deduced with no reference to the law connecting stresses and strains, they will be satisfied by a stress function $F$ such that

$$
X_{x}=\frac{\partial^{2} F}{\partial y^{2}}, \quad Y_{\nu}=\frac{\partial^{2} F}{\partial x^{2}}, \quad X_{\nu}=-\frac{\partial^{2} F}{\partial x \partial y} \text {. }
$$

Substituting Eq. (2) into Eq. (1) and then making use of the compatibility equation

$$
\frac{\partial^{2} e_{x x}}{\partial y^{2}}+\frac{\partial^{2} e_{y y}}{\partial x^{2}}=\frac{\partial^{2} e_{x y}}{\partial x \partial y},
$$

we obtain the following differential equation, satisfied by the stress function $F^{3}$ :

$$
\frac{\partial^{4} F}{\partial x^{4}}+2 K \frac{\partial^{4} F}{\partial x^{2} \partial \eta^{2}}+\frac{\partial^{4} F}{\partial \eta^{4}}=0
$$

where $^{4}$

$$
K=\frac{\left(E_{x} E_{y}\right)^{1 / 2}}{2}\left(\frac{1}{\mu_{x y}}-\frac{2 \sigma_{x y}}{E_{x}}\right)
$$

and

$$
\eta=\epsilon y
$$

with

$$
\epsilon=\left(E_{x} / E_{y}\right)^{1 / 4} .
$$

The solution of Eq. (3) is facilitated by taking $F$ to be of the form

$$
F=R\left[F_{1}(x+i \alpha \eta)+F_{2}(x+i \beta \eta)\right]
$$

where

$$
\alpha=\left[K+\left(K^{2}-1\right)^{1 / 2}\right]^{1 / 2}, \quad \beta=\left[K-\left(K^{2}-1\right)^{1 / 2}\right]^{1 / 2},
$$

$i$ is the imaginary unit, and the letter $R$ means that the real part of the expression in the bracket is to be taken. The functions $F_{1}$ and $F_{2}$ are any analytic functions of the complex variables $x+i \alpha \eta$ and $x+i \beta \eta$, respectively.

${ }^{3}$ For an orthotropic plate like the one discussed here the relation $\sigma_{x y} E_{y}=\sigma_{y x} E_{x}$ holds. See U. S. Forest Products Laboratory Report No. 1503, p. 7.

${ }^{4}$ For wood, $K$ as defined here is probably always greater than 1. 
The stress distribution in an orthotropic plate in a state of plane stress is then obtained by finding a solution to Eq. (3) in the form of Eq. (7), and in addition requiring that the function $F$ satisfies the following relations on the boundary of the plate ${ }^{5}$ :

$$
\frac{d}{d s}\left(\frac{\partial F}{\partial y}\right)=X_{\nu}, \quad \frac{d}{d s}\left(\frac{\partial F}{\partial x}\right)=-Y_{\nu}
$$

where $d s$ denotes an element of the boundary, and $X_{\nu} d s$ and $Y_{\nu} d s$ represent the $x$ and $y$-components of the force per unit thickness of the plate acting on the element of $\operatorname{arc} d s$ from the positive side as indicated by the direction of $\nu$, the normal to the boundary.

A suitable choice of $F$ for the problem considered is

$$
F=R\left[p B\left\{z_{1} \log \left(z_{1}+w_{1}\right)-w_{1}-z_{2} \log \left(z_{2}+w_{2}\right)+w_{2}\right\}\right]
$$

where

$$
\begin{gathered}
z_{1}=x+i \alpha \eta, \quad z_{2}=x+i \beta \eta, \\
w_{1}=\left(z_{1}^{2}+\gamma_{1}^{2}\right)^{1 / 2}, \quad w_{2}=\left(z_{2}^{2}+\gamma_{2}^{2}\right)^{1 / 2}, \\
\gamma_{1}^{2}=b^{2}+\alpha^{2} \epsilon^{2} a^{2}, \quad \gamma_{2}^{2}=b^{2}+\beta^{2} \epsilon^{2} a^{2}, \\
B=\frac{a}{\epsilon\{\beta \arctan (\beta \epsilon \lambda)-\alpha \arctan (\alpha \epsilon \lambda)\}}, \quad \lambda=\frac{a}{b},
\end{gathered}
$$

and $p=P / 2 a d$, the average stress on the cross-section $x=0$, since the plate is taken to be of thickness $d$. To make the functions appearing in Eq. (9) single valued the following relations must be assumed always to hold:

$$
\begin{aligned}
R\left[w_{1}\right] & >0, & R\left[w_{2}\right]>0, \\
-\frac{\pi}{2}<\operatorname{amp}\left(z_{1}+w_{1}\right)<\frac{\pi}{2}, & & -\frac{\pi}{2}<\operatorname{amp}\left(z_{2}+w_{2}\right)<\frac{\pi}{2}
\end{aligned}
$$

By means of Eqs. (2) and (9) the stress components are found to be

$$
\begin{gathered}
\frac{\partial^{2} F}{\partial x^{2}}=Y_{\nu}=R\left[p B\left(\frac{1}{w_{1}}-\frac{1}{w_{2}}\right)\right] \\
\frac{\partial^{2} F}{\partial y^{2}}=X_{x}=R\left[p B\left(\frac{-\alpha^{2} \epsilon^{2}}{w_{1}}+\frac{\beta^{2} \epsilon^{2}}{w_{2}}\right)\right], \\
-\frac{\partial^{2} F}{\partial x \partial y}=X_{\nu}=R\left[p B\left(\frac{-i \alpha \epsilon}{w_{1}}+\frac{i \beta \epsilon}{w_{2}}\right)\right] .
\end{gathered}
$$

The stress component $X_{x}$ as given by Eq. (13) was calculated at several points along the $y$-axis for a plain-sawn plate of Sitka spruce with hyperbolic notches, as in

5S. Timoshenko, Theory of elasticity, McGraw-Hill Co., New York, 1934, p. 179. U. S. Forest Products Laboratory Report No. 1510, p. 7. 
the problem discussed here. The $x$-axis was taken parallel to the grain of the wood. The elastic constants were taken to be:

$$
\begin{aligned}
& E_{x}=1.679 \times 10^{6} \mathrm{lb} . / \text { in. }^{2}, \quad \mu_{x y}=0.112 \times 10^{6} \mathrm{lb} . / \text { in. }^{2}, \\
& E_{y}=0.076 \times 10^{6} \mathrm{lb} . / \text { in. }^{2}, \quad \sigma_{x y}=0.464
\end{aligned}
$$

The results of these calculations for several values of $\lambda$ are given in the table below.

VALUES OF $X_{x}$ FROM EQ. (13)

\begin{tabular}{c|c|c|c|c|c}
\hline \hline \multicolumn{2}{c|}{} & \multicolumn{2}{|c|}{$\lambda=10$} & \multicolumn{2}{|c}{$\lambda=0.1$} \\
\hline$y$ & $X_{x} / p$ & $y$ & $X_{x} / p$ & $y$ & $X_{x} / p$ \\
\hline $0.0 a$ & 0.6373 & $0.0 a$ & 0.6989 & $0.0 a$ & 0.9784 \\
$0.2 a$ & 0.6505 & $0.2 a$ & 0.7142 & $0.2 a$ & 0.9808 \\
$0.4 a$ & 0.6955 & $0.4 a$ & 0.7662 & $0.4 a$ & 0.9884 \\
$0.6 a$ & 0.7971 & $0.6 a$ & 0.8832 & $0.6 a$ & 1.001 \\
$0.8 a$ & 1.064 & $0.8 a$ & 1.180 & $0.8 a$ & 1.020 \\
$1.0 a$ & 30.84 & $1.0 a$ & 3.192 & $1.0 a$ & 1.046 \\
& & & & & \\
\hline
\end{tabular}

It is evident that the value of the stress component $X_{x}$ at $x=0, y=a$ is the maximum stress occurring in the plate in each case. Setting $x=0, y=a$ in Eq. (13), it is found that

$$
\frac{\left(X_{x}\right)_{\max }}{p}=\frac{\epsilon^{2}\left(\beta^{2}-\alpha^{2}\right)}{b} B,
$$

which gives the ratio of the maximum stress to $p$, the average stress on the cross-section $x=0$.

3. Isotropic stress function as the limiting case of the orthotropic stress function. It is of interest to obtain from Eq. (9) the stress function for the corresponding problem for an isotropic material. For an isotropic material $\alpha=\beta=\epsilon=1$, as is shown by Eqs. (4), (6), and (8). For these values, the right side of Eq. (9) becomes indeterminate. Evaluating, it yields

$$
F=R\left[\frac{\left(1+\lambda^{2}\right) a p}{\left(1+\lambda^{2}\right) \arctan \lambda+\lambda}\left\{\frac{a^{2}}{z+w}-i y \log (z+w)\right\}\right]
$$

where

$$
\begin{gathered}
z=x+i y, \\
w=\left(z^{2}+\gamma^{2}\right)^{1 / 2}, \quad \gamma^{2}=a^{2}+b^{2} .
\end{gathered}
$$

To make $F$ single-valued, relations similar to those of Eq. (11) must hold. By letting $z=\gamma z^{\prime}=\gamma\left(x^{\prime}+i y^{\prime}\right)$ in Eq. (16), it is found that

$$
Y_{y}=\frac{\partial^{2} F}{\partial x^{2}}=\frac{1}{\gamma^{2}} \frac{\partial^{2} F}{\partial x^{\prime 2}}=\frac{\partial^{2}}{\partial x^{\prime 2}}\left(\frac{F}{\gamma^{2}}\right)
$$


with similar expressions for the other stress components. If, therefore, in Eq. (16) we replace $z$ by $\gamma z$ and then divide the right side by $\gamma^{2}$, the resulting expression can be used as the stress function. Hence the change to elliptic coordinates, which is usually done by replacing $z$ by $\gamma \sinh (u+i v)$, can be accomplished in Eq. (16) by substituting $\sinh$ $(u+i v)$ for $z$ and then dividing the right side by $\gamma^{2}$. Thus we find the following expression for the stress function in elliptic coordinates:

$$
F=p \frac{\sin v_{0}}{v_{0}+\sin v_{0} \cos v_{0}}\left[y v+\left(\sin ^{2} v_{0}\right) e^{-u} \cos v\right]
$$

where the hyperbola $y^{2} / a^{2}-x^{2} / b^{2}=1$ is given by the particular value, $v_{0}$, of the coordinate $v$. Equation (19) is the stress function for the corresponding problem involving the isotropic plate. ${ }^{6}$

${ }^{6}$ See paper by H. Neuber, Zeitsch. f. Angew. Math. u. Mech. 13, 439 (1933).

\section{BOOK REVIEWS}

Eigenfunction expansions associated with second-order differential equations. By $\cdot \mathrm{E}$. C. Titchmarsh. Oxford, at the Clarendon Press, 1946. 175 pp. $\$ 7.00$.

The subject matter of this book is composed of the analysis required to develop and justify the expansion of an arbitrary function $f(x)$ in the eigenfunctions of the differential equation $y^{\prime \prime}+[\lambda-q(x)] y=0$. The classical theory is disposed of in Chapter 1 and the remainder of the book is largely devoted to those situations where $q(x)$ is singular at an end-point of the region and/or those where $f(x)$ is defined over an infinite or semi-infinite domain. In Chapter 4 one finds as examples the expansion formulae for Hermite, Bessel, Legendre, Sonine, Laguerre, and hypergeometric functions. The latter chapters deal with the distribution of the eigenvalues, the nature of the spectrum, and with questions of convergence and summability.

The book is intended primarily for the mathematician, but its interest and value to the physicist and engineer should be great.

G. F. CArrier

Modern operational calculus with applications in technical mathematics. By $\mathrm{N}$. W. McLachlan. MacMillan and Co., Ltd., London, 1948. xiv + 218 pp. $\$ 5.00$.

This introduction to the theory of the Laplace Transform is intended as a guide to those interested in the application of this transform to engineering problems. However, the questions of rigor are treated in more detail than is customary in texts with this purpose. In fact, nearly one third of the book consists of appendices dealing with such items as convergence questions. In Chapters 1 and 2 the transform is defined and the fundamental theorems and identities are deduced. In Chapters 3 and 4, the solution by transform methods of ordinary and partial linear differential equations is discussed. These and the subsequent chapters which, for the most part, are concerned with integral evaluation contain many examples drawn largely from electric and acoustic problems. It is regrettable that no reference has been made to the role of this transform in solving stability problems.

The book should be especially useful to engineers whose background is essentially non-mathematical since the points of rigor discussed appear to have been selected for just such an audience. 